\title{
CONVEX HULL PROPERTY AND MAXIMUM PRINCIPLE FOR FINITE ELEMENT MINIMISERS OF GENERAL CONVEX FUNCTIONALS
}

\author{
LARS DIENING, CHRISTIAN KREUZER, AND SEBASTIAN SCHWARZACHER
}

\begin{abstract}
The convex hull property is the natural generalization of maximum principles from scalar to vector valued functions. Maximum principles for finite element approximations are often crucial for the preservation of qualitative properties of the respective physical model. In this work we develop a convex hull property for $\mathbb{P}_{1}$ conforming finite elements on simplicial non-obtuse meshes. The proof does not resort to linear structures of partial differential equations but directly addresses properties of the minimiser of a convex energy functional. Therefore, the result holds for very general nonlinear partial differential equations including e.g. the $p$-Laplacian and the mean curvature problem. In the case of scalar equations the introduce techniques can be used to prove standard discrete maximum principles for nonlinear problems. We conclude by proving a strong discrete convex hull property on strictly acute triangulations.
\end{abstract}

\section{INTRODUCTION}

Let the bounded polyhedral domain $\Omega \subset \mathbb{R}^{n}, n \geqslant 2$, be partitioned by a conforming simplicial triangulation $\mathcal{T}$. We denote by $\mathbb{V}(\mathcal{T})^{m}, m \in \mathbb{N}$, the finite element space of $m$-dimensional vector valued, continuous, and piece wise affine functions over $\mathcal{T}$ and by $\mathbb{V}_{0}(\mathcal{T})^{m} \subset \mathbb{V}(\mathcal{T})^{m}$ the subspace of functions with zero boundary values. For $\boldsymbol{G} \in \mathbb{V}(\mathcal{T})^{m}$ we consider minimising problems of the form

$$
\mathcal{J}(\boldsymbol{V}):=\int_{\Omega} F(x,|\nabla \boldsymbol{V}(x)|) \mathrm{d} x \rightarrow \min \quad \text { in } \boldsymbol{G}+\mathbb{V}_{0}(\mathcal{T})^{m} .
$$

We suppose that (1.1) admits a unique minimiser and that the mapping $\Omega \times \mathbb{R} \geqslant \ni$ $(x, y) \mapsto F(x, y) \in \mathbb{R}$ is monotone in its second argument; for the precise assumptions see Section 2.2

The following theorem is the main result of this work.

Theorem 1.1 (Convex hull property). Let the triangulation $\mathcal{T}$ be non-obtuse, i.e., the angle between any two sides of any simplex $T \in \mathcal{T}$ is less or equal $\pi / 2$.

Then the unique minimiser $\boldsymbol{U} \in \boldsymbol{G}+\mathbb{V}_{0}(\mathcal{T})^{m}$ of 1.1 satisfies

$$
\boldsymbol{U}(\Omega) \subset \operatorname{conv} \text { hull }(\boldsymbol{U}(\partial \Omega))=\operatorname{conv} \text { hull }(\boldsymbol{G}(\partial \Omega)) .
$$

This is the discrete analogue of the so called convex hull property of vector valued minimisers; see [DLM98, BF02. Note that the convex hull property is the generalization of the maximum principle to the vector valued case. Indeed, if $m=1$, then the convex hull property reads as

$$
U(\Omega)=[\min U(\partial \Omega), \max U(\partial \Omega)] .
$$

2010 Mathematics Subject Classification. 65N30 and 35J60 and 35J92 and 35J93.

Key words and phrases. discrete maximum principle and strong discrete maximum principle and finite elements and nonlinear pde and mean curvature and p-Laplace. 
The problem above is presented as a minimising problem. If the energy $\mathcal{J}$ is Fréchet differentiable, then (1.1) becomes equivalent to finding a solution $\boldsymbol{U} \in$ $\boldsymbol{G}+\mathbb{V}_{0}(\mathcal{T})$ of the Euler-Lagrange system

$$
\langle D \mathcal{J}(\boldsymbol{U}), \boldsymbol{H}\rangle=\int_{\Omega} a(x,|\nabla \boldsymbol{U}(x)|) \nabla \boldsymbol{U}: \nabla \boldsymbol{H} \mathrm{d} x=0 \quad \text { for all } \boldsymbol{H} \in \mathbb{V}_{0}(\mathcal{T}),
$$

where $a(x, t)=F_{t}(x, t) / t$. Consequently, Theorem 1.1 applies to a large class of non-linear partial differential equations.

The research on discrete maximum principles can be traced back to the early paper of Ciarlet and Raviart CR73] in the nineteen seventies. Provided a non-obtuse triangulation, they established a discrete maximum principle for scalar valued linear second order elliptic problems on a two dimensional domain. This result was generalized to three dimensional domains in KQ95. For the Laplacian, the above mesh condition can be replaced by a weaker, so called Delaunay condition. This condition only requires that the sum of any pair of angles opposite a common side is less or equal $\pi$; see [SF73, Let92]. Drăgănescu, Dupont, and Scott DDS05 analyzed the failure of the discrete maximum principle when the Delaunay condition is violated. However, according to [RS82, KKN01] a discrete maximum principle may hold even if both angles in such a pair are greater than $\pi / 2$ introducing additional restrictions involving a larger neighborhood. Some of these concepts are generalized to anisotropic diffusion problems in [LH10, Hua11].

Recently, discrete maximum principles are proved for quasi-linear partial differential equations [KK05a, WZ12, which means in the case of 1.2 that $0<\lambda \leqslant$ $a(x, t) \leqslant \Lambda<\infty$ for all $x, t \in \Omega \times \mathbb{R}$. Jüngel and Unterreitner [JU05] proved a discrete maximum principle for partial differential equations with non-linear lowest order term.

All aforementioned approaches are based on the ellipticity and continuity of the problems in order to allow for a Hilbert space setting. In particular, beside WZ12, JU05, they rely on the inversion of so called $M$-Matrices. As a consequence the results are basically restricted to energy functionals with quadratic growth.

In contrast, our approach is based on a projection into a suitable convex set and direct properties of the energy functional. Therefore, it does not rely on linear structures and directly applies to general convex non-linear, vector valued problems. As a drawback, we need point-wise (resp. element-wise) properties of the finite element functions and therefore cannot weaken the mesh restrictions to a Delaunay type condition.

The plan of the paper is as follows. In Section 2 we introduce the finite element framework and provide precise assumptions on the energy functional from (1.1). In Section 3 we prove the discrete convex hull property, Theorem 1.1. We close the article in Section 4 discussing several applications and extensions of the introduced theory. In particular, we prove a maximum principle for non-linear scalar partial differential equations with non-positive right hand side and show that the $p$-Laplace, as well as the mean curvature problem fit into the presented theory. Moreover, we show a discrete maximum principle for non-linear problems with lower order terms using mass-lumping. Finally, we define and verify a strong convex hull property, which generalizes the strong maximum principle of scalar valued problems.

\section{Problem Setting}

In this section we shall introduce the finite element setting and formulate the precise assumptions on the non-linear energy. For the sake of presentation we denote the Euclidean norms in $\mathbb{R}^{n}, n \geqslant 2, \mathbb{R}^{m}, m \in \mathbb{N}$, as well as the absolute value in $\mathbb{R}$ by $|\cdot|$. Furthermore, for $\boldsymbol{A}=\left[A_{i j}\right]_{\substack{i=1, \ldots, n \\ j=1, \ldots, m}}, \boldsymbol{B}=\left[B_{i j}\right]_{\substack{i=1, \ldots, n \\ j=1, \ldots, m}} \in \mathbb{R}^{n \times m}$ we define the 
inner product $\boldsymbol{A}: \boldsymbol{B}:=\sum_{\substack{i=1, \ldots, n \\ j=1, \ldots, m}} A_{i j} B_{i j}$ and denote also by $|\boldsymbol{A}|$ the corresponding Frobenius norm of $\boldsymbol{A}$.

2.1. Finite Element Framework. Let $\mathcal{T}$ be a conforming partition of the polyhedral domain $\Omega \subset \mathbb{R}^{n}$ into closed $n$-simplexes. To be more precise, we have

$$
\bar{\Omega}=\bigcup\{T \mid T \in \mathcal{T}\}
$$

and the intersection of two different elements in $\mathcal{T}$ is either empty or a complete $k$-dimensional sub-simplex, $0 \leqslant k<n$. Let $\mathbb{P}_{1}(T)$ be the space of affine linear functions on $T \in \mathcal{T}$ and define the space of first order Lagrange finite elements by

$$
\mathbb{V}(\mathcal{T}):=\left\{V \in C(\bar{\Omega}): V_{\mid T} \in \mathbb{P}_{1}(T) \text { for all } T \in \mathcal{T}\right\} .
$$

This space is spanned by the so called nodal Lagrange basis. To be more precise, let $\mathcal{N}$ be the set of vertices of all elements in $\mathcal{T}$ and let $\mathcal{N}_{0}:=\mathcal{N} \cap \Omega$ be the subset of vertices inside $\Omega$. For $z \in \mathcal{N}$ the corresponding Lagrange hat function $\phi_{z}$ is uniquely defined by $\phi_{z}(y)=\delta_{z y}$ for all $y \in \mathcal{N}$, where we make use of the Kronecker symbol, i.e., $\delta_{z y}=1$ whenever $z=y$ and $\delta_{z y}=0$ else. We denote the set of element sides of $\mathcal{T}$ by $\mathcal{S}$. For two sides of $S_{1}, S_{2} \in \mathcal{S}$ of a simplex $T \in \mathcal{T}$ we define the angle between $S_{1}$ and $S_{2}$ by $\angle\left(S_{1}, S_{2}\right):=\pi-\angle\left(\boldsymbol{n}_{1}, \boldsymbol{n}_{2}\right)$, where $\angle\left(\boldsymbol{n}_{1}, \boldsymbol{n}_{2}\right)$ is the angle in between the normals $\boldsymbol{n}_{1}$ and $\boldsymbol{n}_{2}$ pointing inside $T$ from $S_{1}$ and $S_{2}$, respectively.

In the following we introduce non-obtuse meshes, which play a crucial role in the analysis of discrete maximum principles; see e.g. [CR73, KQ95, DDS05].

Definition 2.1 (non-obtuseness). A simplex $T \in \mathcal{T}$ is called non-obtuse if the angles between any two of its sides are less or equal $\frac{\pi}{2}$.

The conforming triangulation $\mathcal{T}$ of $\Omega$ is called non-obtuse if all simplexes $T \in \mathcal{T}$ are non-obtuse.

We remark that in higher dimensions $n \geqslant 3$ the construction and refinement of non-obtuse partitions is a non trivial task. The interested reader is e.g. referred to KQ95, KK05b, KK11.

One can characterize non-obtuse meshes by means of the nodal Lagrange basis functions. Indeed, observing that the gradient of a Lagrange basis function $\phi_{z}$, $z \in \mathcal{N} \cap T$ on a simplex $T \in \mathcal{T}$ points in the direction of the normal of the side opposite $z$, one can easily show that the following condition on the basis functions is equivalent to the geometric mesh property in Definition 2.1 compare also with CR73, KQ95.

Proposition 2.2. An n-simplex $T \in \mathcal{T}$ is non-obtuse if and only if

$$
\nabla \phi_{z \mid T} \cdot \nabla \phi_{y \mid T} \leqslant 0 \quad \text { for all } z, y \in T \cap \mathcal{N} \text { with } z \neq y \text {. }
$$

A direct consequence is the following corollary.

Corollary 2.3. Let $\mathcal{T}$ be a conforming triangulation of $\Omega$. Then $\mathcal{T}$ is non-obtuse if and only if

$$
\nabla \phi_{z} \cdot \nabla \phi_{y} \leqslant 0 \quad \text { a. e. in } \Omega \quad \text { for all } z, y \in \mathcal{N} \text { with } z \neq y .
$$

The subspace of functions in $\mathbb{V}(\mathcal{T})$ vanishing at the boundary $\partial \Omega$ is denoted by

$$
\mathbb{V}_{0}(\mathcal{T}):=\left\{V \in \mathbb{V}(\mathcal{T}): V_{\mid \partial \Omega}=0\right\}=\operatorname{span}\left\{\phi_{z}: z \in \mathcal{N}_{0}\right\}
$$

For $m \in \mathbb{N}$, the $m$-dimensional vector valued finite element spaces corresponding to $\mathbb{V}(\mathcal{T})$ and $\mathbb{V}_{0}(\mathcal{T})$ are given by

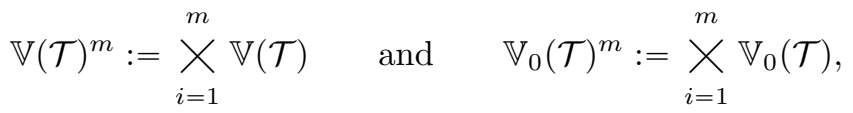


respectively. Clearly, for $\boldsymbol{G} \in \mathbb{V}(\mathcal{T})^{m}$, the affine subspace of functions that coincide with $\boldsymbol{G}$ on $\partial \Omega$ is $\boldsymbol{G}+\mathbb{V}_{0}(\mathcal{T})^{m}$. For $\omega \subset \bar{\Omega}$ we define $\boldsymbol{V}(\omega):=\{\boldsymbol{V}(x): x \in \omega\} \subset \mathbb{R}^{m}$ as the set of function values of $\boldsymbol{V}$ on $\omega$.

2.2. Energy Minimisation. In this section we formulate standard conditions on the energy $\mathcal{J}$ in order to guarantee the unique solvability of $(1.1)$. To this end we first ensure that $\mathcal{J}(\boldsymbol{V})$ is well defined for all $\boldsymbol{V} \in \mathbb{V}(\mathcal{T})^{m}$ assuming that $F: \Omega \times \mathbb{R}_{\geqslant} \rightarrow$ $\mathbb{R}$ defines a Nemyckii operator $\mathcal{F}: \mathbb{V}(\mathcal{T})^{m} \rightarrow L^{1}(\Omega)$ setting

$$
(\mathcal{F} \boldsymbol{V})(x) \mapsto F(x,|\nabla \boldsymbol{V}(x)|), \quad x \in \Omega .
$$

This can be achieved by the following conditions.

Assumption 2.4 (Nemyckii Operator). We assume that $F: \Omega \times \mathbb{R}_{\geqslant} \rightarrow \mathbb{R}$

(1) is a Carathéodory function, i.e., $F(\cdot, t)$ is measurable in $\Omega$ for each $t \in \mathbb{R}_{\geqslant}$ and $F(x, \cdot)$ is continuous on $\mathbb{R}_{\geqslant}$for almost every $x \in \Omega$;

(2) satisfies the growth condition

$$
|F(x, t)| \leqslant \alpha(x)+\gamma(t), \quad(x, t) \in \Omega \times \mathbb{R}_{\geqslant},
$$

for some $\alpha \in L^{1}(\Omega)$ and continuous $\gamma: \mathbb{R}_{\geqslant} \rightarrow \mathbb{R}_{\geqslant}$.

Proposition 2.5. Suppose that $F: \Omega \times \mathbb{R}_{\geqslant} \rightarrow \mathbb{R}$ satisfies Assumptions 2.4. Then the energy functional $\mathcal{J}: \mathbb{V}(\mathcal{T})^{m} \rightarrow L^{1}(\Omega)$ defined in $(1.1)$ is continuous.

Proof. Let $\left\{\boldsymbol{V}_{k}\right\}_{k \in \mathbb{N}} \subset \mathbb{V}(\mathcal{T})^{m}, \boldsymbol{V} \in \mathbb{V}(\mathcal{T})^{m}$ such that $\boldsymbol{V}_{k} \rightarrow \boldsymbol{V}$ in $\mathbb{V}(\mathcal{T})^{m}$ as $k \rightarrow \infty$. The space $\mathbb{V}(\mathcal{T})^{m}$ is finite dimensional and hence all norms on $\mathbb{V}(\mathcal{T})^{m}$ are equivalent. Therefore, we can conclude that the sequence $\left\{\boldsymbol{V}_{k}\right\}_{k \in \mathbb{N}}$ is uniformly bounded in $L^{\infty}(\Omega)^{m}$. Assumption 2.4 (2) thus implies for some $C>0$ that $\left|F\left(\cdot, \boldsymbol{V}_{k}(\cdot)\right)\right| \leqslant \alpha(\cdot)+C \in L^{1}(\Omega)$ and from Assumption 2.4 (1) it follows that $F\left(\cdot, \boldsymbol{V}_{k}(\cdot)\right) \rightarrow F(\cdot, \boldsymbol{V}(\cdot))$ almost everywhere in $\Omega$. Hence the claim is a consequence of Lebesgue's dominated convergence theorem.

The following conditions are linked to the existence of a unique minimiser of $\mathcal{J}$.

Assumption 2.6. We assume that $F: \Omega \times \mathbb{R}_{\geqslant} \rightarrow \mathbb{R}$ is strictly convex in its second argument, i.e., it holds for all $\theta \in(0,1)$ and $s, t \geqslant 0$ with $s \neq t$, that

$$
F(\cdot, \theta s+(1-\theta) t)<\theta F(\cdot, s)+(1-\theta) F(\cdot, t) \quad \text { a.e. in } \Omega .
$$

Moreover, assume that $F$ satisfies the coercivity condition

$$
F(\cdot, t) \geqslant g(t) \quad \text { for all } t \geqslant 0 \text { a.e. in } \Omega,
$$

with some continuous monotone function $g: \mathbb{R}_{\geqslant} \rightarrow \mathbb{R}$ such that $g(t) \rightarrow \infty$ as $t \rightarrow \infty$.

Now we are prepared to prove the existence of a unique solution to 1.1 .

Proposition 2.7. Let $\boldsymbol{G} \in \mathbb{V}(\mathcal{T})^{m}$ and suppose Assumptions 2.4 and 2.6. Then there exists a unique $\boldsymbol{U} \in \boldsymbol{G}+\mathbb{V}_{0}(\mathcal{T})^{m}$ such that

$$
\mathcal{J}(\boldsymbol{U})=\min \left\{\mathcal{J}(\boldsymbol{V}): \boldsymbol{V} \in \boldsymbol{G}+\mathbb{V}_{0}(\mathcal{T})^{m}\right\}
$$

Proof. The proof of this assertion is fairly standard. However, for the sake of a selfconsistent presentation we sketch the proof here. Thanks to $2.2 \mathrm{~b})$ it follows that the energy functional is bounded from below and that an infimal sequence $\left\{\boldsymbol{V}_{k}\right\}_{k \in \mathbb{N}} \subset$ $\boldsymbol{G}+\mathbb{V}_{0}(\mathcal{T})^{m}$ is norm-wise uniformly bounded by some constant $C>0$. Since $\mathbb{V}(\mathcal{T})^{m}$ is of finite dimension, $\left\{\boldsymbol{V}_{k}\right\}_{k \in \mathbb{N}}$ is pre-compact in $\mathbb{V}(\mathcal{T})^{m}$. Consequently, there exists a converging subsequence $\boldsymbol{V}_{k_{\ell}} \rightarrow \boldsymbol{U}$ for some $\boldsymbol{U} \in \boldsymbol{G}+\mathbb{V}_{0}(\mathcal{T})^{m}$ as $\ell \rightarrow \infty$. By the continuity of $\mathcal{J}$, Proposition 2.5 it follows that $\boldsymbol{U}$ is a minimiser of $\mathcal{J}$. Finally, the strict convexity 2.2a implies the uniqueness of the minimiser. 


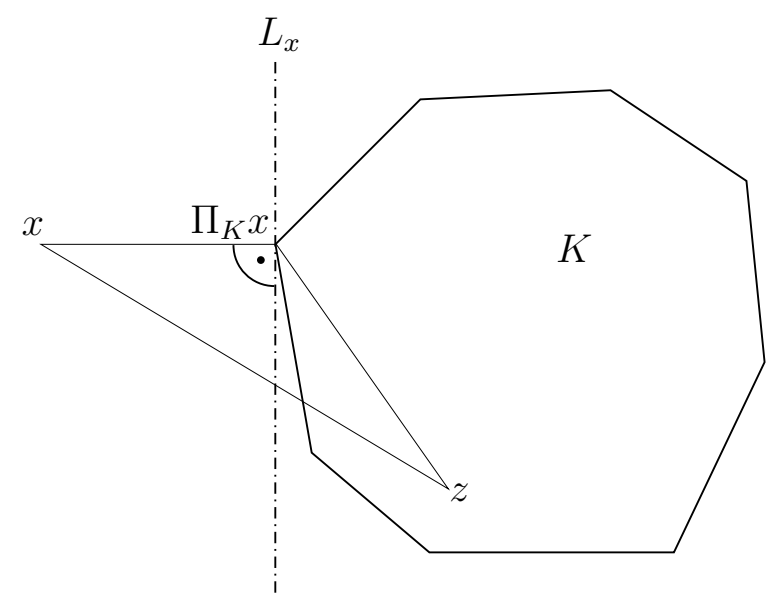

Figure 1. The projection $\Pi_{K}$

The following assumption is crucial in order to prove the discrete maximum principle (Theorem 1.1).

Assumption 2.8 (Monotonicity). We assume that the mapping $F(x, \cdot): \mathbb{R}_{\geqslant} \rightarrow \mathbb{R}$ is monotone increasing for almost every $x \in \Omega$.

Note that $\mathbb{V}(\mathcal{T}) \subset L^{\infty}(\Omega)$ is finite dimensional. Therefore, in Assumptions 2.4 and 2.6 we do not need to resort to common growth or coercivity conditions like $\gamma(t)=c|t|^{s}$ or $g(t)=c|t|^{r}$ for some $c>0$ and $s>1$. As a consequence, the presented theory can be applied e.g. to the mean curvature problem; compare also with Remark 4.3 below.

\section{Proof of the Discrete Convex Hull Property, Theorem 1.1}

In the following let $K \subset \mathbb{R}^{m}$ denote a convex, closed subset. We denote by $\Pi_{K}: \mathbb{R}^{m} \rightarrow K$ the orthogonal projection with respect to the Euclidean inner product of $\mathbb{R}^{m}$. In other words

$$
\Pi_{K} x:=\operatorname{argmin}_{y \in K}|x-y| .
$$

The next lemma is an immediate consequence of the convexity of the set $K$.

Lemma 3.1. Let $K \subset \mathbb{R}^{m}$ be a closed and convex. Then for all $x \in \mathbb{R}^{m}$ and $z \in K$ we have

$$
\left(x-\Pi_{K} x\right) \cdot\left(z-\Pi_{K} x\right) \leqslant 0 .
$$

Proof. If $x \in K$, then $x=\Pi_{K} x$ and the estimate is obvious. Let us assume that $x \notin K$. We denote by $L_{x}$ the hyper-plane which touches $K$ in $\Pi_{K} x$ and is orthogonal to $x-\Pi_{K} x$. Since $K$ is convex, $L_{x}$ separates $K$ from $x$. This implies that the angle between $\left(\Pi_{K} x-x\right)$ and $\left(\Pi_{K} x-z\right)$ is greater or equal $\pi / 2$; compare with Figure 1 . This proves the assertion.

We define a projection operator $\mathcal{P}_{K}: \mathbb{V}(\mathcal{T})^{m} \rightarrow \mathbb{V}(\mathcal{T})^{m}$ with $\left(\mathcal{P}_{K} \boldsymbol{V}\right)(\Omega) \subset K$ by setting

$$
\left(\mathcal{P}_{K} \boldsymbol{V}\right)(z):=\Pi_{K} \boldsymbol{V}(z), \quad z \in \mathcal{N} .
$$

We next list some elementary properties of this projection operator. In particular, since $K$ is convex and $\mathbb{V}(\mathcal{T})^{m}$ contains only piecewise linear functions over $\mathcal{T}$, we have

$$
\mathcal{P}_{K} \boldsymbol{V}: \Omega \rightarrow K \quad \text { for all } \boldsymbol{V} \in \mathbb{V}(\mathcal{T})^{m}
$$


If $\boldsymbol{G}(\partial \Omega) \subset K$, for some $\boldsymbol{G} \in \mathbb{V}(\mathcal{T})^{m}$, it follows that the boundary values are preserved, i.e.,

$$
\mathcal{P}_{K} \boldsymbol{V} \in \boldsymbol{G}+\mathbb{V}_{0}(\mathcal{T})^{m} \quad \text { for all } \boldsymbol{V} \in \boldsymbol{G}+\mathbb{V}_{0}(\mathcal{T})^{m}
$$

The following property of $\mathcal{P}_{K}$ is the key estimate in the proof of the convex hull property.

Lemma 3.2. Let $\mathcal{T}$ be a non-obtuse conforming triangulation of $\Omega$ and let $K \subset \mathbb{R}^{m}$ be a closed and convex set. Then for $\boldsymbol{V} \in \mathbb{V}(\mathcal{T})^{m}$ we have

$$
\nabla \boldsymbol{V}: \nabla \mathcal{P}_{K} \boldsymbol{V} \geqslant\left|\nabla \mathcal{P}_{K} \boldsymbol{V}\right|^{2} \quad \text { a.e. in } \Omega
$$

and

$$
|\nabla \boldsymbol{V}| \geqslant\left|\nabla \mathcal{P}_{K} \boldsymbol{V}\right| \quad \text { a.e. in } \Omega \text {. }
$$

Proof. We prove the estimates on a fixed $n$-simplex $T \in \mathcal{T}$. Let $z_{0}, \ldots, z_{n}$ denote the vertices of $T$ and let $\phi_{0}, \ldots, \phi_{n}$ be the corresponding local Lagrange basis functions. Then

$$
\boldsymbol{V}_{\mid T}=\sum_{i=0}^{n} \boldsymbol{V}\left(z_{i}\right) \phi_{i \mid T}
$$

and

$$
\nabla \boldsymbol{V}_{\mid T}: \nabla\left(\mathcal{P}_{K} \boldsymbol{V}\right)_{\mid T}=\sum_{i=0}^{n} \sum_{k=0}^{n}\left(\boldsymbol{V}\left(z_{i}\right) \cdot \Pi_{K} \boldsymbol{V}\left(z_{k}\right)\right)\left(\nabla \phi_{i \mid T} \cdot \nabla \phi_{k \mid T}\right)
$$

Using $\nabla \phi_{0}+\nabla \phi_{1}+\cdots+\nabla \phi_{n}=\nabla 1=0$ on $T$ we conclude

$$
\nabla \boldsymbol{V}_{\mid T}: \nabla\left(\mathcal{P}_{K} \boldsymbol{V}\right)_{\mid T}=\sum_{i=0}^{n} \sum_{k \neq i} \boldsymbol{V}\left(z_{i}\right) \cdot\left(\Pi_{K} \boldsymbol{V}\left(z_{k}\right)-\Pi_{K} \boldsymbol{V}\left(z_{i}\right)\right) \nabla \phi_{i \mid T} \cdot \nabla \phi_{k \mid T}
$$

Lemma 3.1 yields

$$
\left(\boldsymbol{V}\left(z_{i}\right)-\Pi_{K} \boldsymbol{V}\left(z_{i}\right)\right) \cdot\left(\Pi_{K} \boldsymbol{V}\left(z_{k}\right)-\Pi_{K} \boldsymbol{V}\left(z_{i}\right)\right) \leqslant 0,
$$

and thus

$$
\boldsymbol{V}\left(z_{i}\right) \cdot\left(\Pi_{K} \boldsymbol{V}\left(z_{k}\right)-\Pi_{K} \boldsymbol{V}\left(z_{i}\right)\right) \leqslant \Pi_{K} \boldsymbol{V}\left(z_{i}\right) \cdot\left(\Pi_{K} \boldsymbol{V}\left(z_{k}\right)-\Pi_{K} \boldsymbol{V}\left(z_{i}\right)\right) .
$$

Since $T$ is non-obtuse we have by Proposition 2.2 that $\nabla \phi_{i \mid T} \cdot \nabla \phi_{k \mid T} \leqslant 0$ for all $i \neq k$. Consequently, we arrive at

$$
\begin{aligned}
\nabla \boldsymbol{V}_{\mid T}: \nabla\left(\mathcal{P}_{K} \boldsymbol{V}\right)_{\mid T} & \geqslant \sum_{i=0}^{n} \sum_{k \neq i}\left(\Pi_{K} \boldsymbol{V}\left(z_{i}\right) \cdot\left(\Pi_{K} \boldsymbol{V}\left(z_{k}\right)-\Pi_{K} \boldsymbol{V}\left(z_{i}\right)\right)\left(\nabla \phi_{i \mid T} \cdot \nabla \phi_{k \mid T}\right)\right. \\
& =\sum_{i=0}^{n} \sum_{k=0}^{n}\left(\Pi_{K} \boldsymbol{V}\left(z_{i}\right) \cdot \Pi_{K} \boldsymbol{V}\left(z_{k}\right)\right)\left(\nabla \phi_{i \mid T} \cdot \nabla \phi_{k \mid T}\right) \\
& =\nabla\left(\mathcal{P}_{K} \boldsymbol{V}\right)_{\mid T}: \nabla\left(\mathcal{P}_{K} \boldsymbol{V}\right)_{\mid T}=\left|\nabla\left(\mathcal{P}_{K} \boldsymbol{V}\right)_{\mid T}\right|^{2} .
\end{aligned}
$$

This proves the first claim.

Thanks to the Cauchy-Schwarz inequality, the second estimate is an immediate consequence of the first one.

Lemma 3.3. Let $\mathcal{T}$ be a non-obtuse conforming triangulation of $\Omega$ and let $F$ : $\Omega \times \mathbb{R}_{\geqslant} \rightarrow \mathbb{R}$ satisfy Assumptions 2.4, 2.6, and 2.8. For $\boldsymbol{G} \in \mathbb{V}(\mathcal{T})^{m}, m \in \mathbb{N}$, let $K \subset \mathbb{R}^{m}$ be a convex and closed set such that $\boldsymbol{G}(\partial \Omega) \subset K$. Then the uniquely defined solution $\boldsymbol{U} \in \boldsymbol{G}+\mathbb{V}_{0}(\mathcal{T})^{m}$ of 11.1 satisfies

$$
\boldsymbol{U}(\Omega) \subset K \text {. }
$$


Proof. It follows from Proposition 2.7 that problem 1.1 has a unique solution $\boldsymbol{U} \in \boldsymbol{G}+\mathbb{V}_{0}(\mathcal{T})^{m}$. Since $K$ is a closed convex set we conclude from Assumption 2.8 and Lemma 3.2 that

$$
\mathcal{J}(\boldsymbol{U}) \geqslant \mathcal{J}\left(\mathcal{P}_{K} \boldsymbol{U}\right)
$$

It follows by $(3.3 \mathrm{~b})$ that $\mathcal{P}_{K} \boldsymbol{U} \in \boldsymbol{G}+\mathbb{V}_{0}(\mathcal{T})^{m}$. Since $\boldsymbol{U}$ is the unique minimiser of $\mathcal{J}$ in $\boldsymbol{G}+\mathbb{V}_{0}(\mathcal{T})^{m}$ we conclude $\mathcal{P}_{K} \boldsymbol{U}=\boldsymbol{U}$. The desired assertion is then a consequence of $3.3 \mathrm{~b}$.

From this result the discrete maximum principle, Theorem 1.1, follows as a corollary.

Theorem 1.1. The convex set $K:=$ conv hull $(\boldsymbol{G}(\partial \Omega)) \subset \mathbb{R}^{m}$ satisfies all conditions of Lemma 3.3 .

Remark 3.4. We emphasize, that the proof of the convex hull property (Theorem 1.1) does not directly resort to Assumptions 2.4 and 2.6. which are only used in Proposition 2.7 to ensure the existence of a solution. In fact, a unique minimiser $\boldsymbol{U} \in \boldsymbol{G}+\mathbb{V}_{0}(\mathcal{T})$ of (1.1) satisfies the convex hull property (Theorem 1.1) if Assumption 2.8 holds. The uniqueness of the minimiser is usually a consequence of the strict convexity of the energy.

\section{Applications and Extensions}

In this section we apply the above techniques to some particular exemplary problems and extend Theorem 1.1 in several ways.

4.1. The $p$-Laplace Problem. This section is concerned with the so-called $p$ Laplace operator. We verify that $p$-harmonic functions fit into the framework of Section 2 and then prove a discrete maximum principle for scalar solutions of a $p$-Laplace problem with non-positive right-hand side. To this end let $\mathcal{T}$ be a conforming triangulation of the $n$-dimensional domain $\Omega$ with polyhedral boundary, $\boldsymbol{G} \in \mathbb{V}(\mathcal{T})^{m}, m \in \mathbb{N}$, and $p, q \in(1, \infty)$ with $\frac{1}{p}+\frac{1}{q}=1$. It is well known, that

$$
\int_{\Omega}|\nabla \boldsymbol{U}|^{p-2} \nabla \boldsymbol{U}: \nabla \boldsymbol{V} \mathrm{d} x=0 \quad \text { in } \Omega
$$

uniquely determines a function $\boldsymbol{U} \in \boldsymbol{G}+\mathbb{V}_{0}(\mathcal{T})^{m}$. The equivalent minimising problem reads as

$$
\mathcal{J}(\boldsymbol{V}):=\int_{\Omega} \frac{1}{p}|\nabla \boldsymbol{V}|^{p} \mathrm{~d} x \rightarrow \min \quad \text { in } \boldsymbol{G}+\mathbb{V}_{0}(\mathcal{T}) .
$$

It can easily be verified that the operator

$$
F(x, t):=\frac{1}{p}|t|^{p}, \quad t \geqslant 0
$$

satisfies Assumptions 2.4, 2.6, and 2.8. Consequently, if $\mathcal{T}$ is non-obtuse, all conditions of Theorem 1.1 are satisfied.

For $m=1$ and $f \in L^{q}(\Omega)$ we investigate the non-homogeneous $p$-Laplace problem

$$
\begin{aligned}
\int_{\Omega}|\nabla U|^{p-2} \nabla U \cdot \nabla V \mathrm{~d} x & =\int_{\Omega} f V \mathrm{~d} x & & \text { in } \Omega \\
U & =G & & \text { on } \partial \Omega .
\end{aligned}
$$

The unique solution $U \in G+\mathbb{V}_{0}(\mathcal{T})$ is the minimiser of the energy

$$
\mathcal{J}_{f}(V):=\int_{\Omega} \frac{1}{p}|\nabla V|^{p}-f V \mathrm{~d} x, \quad V \in G+\mathbb{V}_{0}(\mathcal{T})
$$


Since the integrand depends not only on the gradient of the function but also on the function value itself, we can not directly apply Theorem 1.1. However, by modifying the arguments we can prove the following result.

Theorem 4.1. Let the conforming triangulation $\mathcal{T}$ of $\Omega$ be non-obtuse and let $f \leqslant 0$ in $\Omega$.

Then the unique solution $U \in G+\mathbb{V}_{0}(\mathcal{T})$ of 4.2 satisfies

$$
\max U(\Omega) \leqslant \max U(\partial \Omega) .
$$

Proof. We define the closed convex set

$$
K:=(-\infty, \max U(\partial \Omega)] \supset \operatorname{conv} \text { hull }(U(\partial \Omega)) .
$$

Hence the projection operator $\mathcal{P}_{K}: \mathbb{V}(\mathcal{T}) \rightarrow \mathbb{V}(\mathcal{T})$, defined in 3.2 satisfies $(3.3)$, i.e., we have that $\mathcal{P}_{K} U \in G+\mathbb{V}_{0}(\mathcal{T})$. Clearly the mapping $(x, t) \mapsto F(x, t):=\frac{1}{p} t^{p}$, $(x, t) \in \Omega \times \mathbb{R}_{\geqslant}$, satisfies all conditions in Section 2.2. In particular, it satisfies Assumption 2.8, i.e., it is monotone increasing in its second argument. Therefore, we obtain by Lemma 3.2 that

$$
\int_{\Omega} \frac{1}{p}\left|\nabla \mathcal{P}_{K} U\right|^{p} \mathrm{~d} x \leqslant \int_{\Omega} \frac{1}{p}|\nabla U|^{p} \mathrm{~d} x .
$$

Moreover, by means of (3.1) and the particular choice of the convex set $K$, we have

$$
\mathcal{P}_{K} U(x)=\min \{U(x), \max U(\partial \Omega)\} \leqslant U(x), \quad \text { for all } x \in \Omega .
$$

Hence the assumption $f \leqslant 0$ in $\Omega$ implies

$$
\int_{\Omega} f \mathcal{P}_{K} U \mathrm{~d} x \geqslant \int_{\Omega} f U \mathrm{~d} x
$$

Combining these estimates yields

$$
\mathcal{J}\left(\mathcal{P}_{K} U\right) \leqslant \mathcal{J}(U)
$$

Since $U$ is the unique minimiser we arrive with $(3.3)$ at $U(\Omega)=\left(\mathcal{P}_{K} U\right)(\Omega) \subset K$. This proves the assertion.

Remark 4.2 (Orlicz functions). The p-Laplace operator often serves as a prototype for a much larger class of non-linear operators: Indeed, if we have a continuous, strictly convex and monotone function $\psi: \mathbb{R}_{\geqslant} \rightarrow \mathbb{R}_{\geqslant}$, then the non-linear function $F(x, t):=\psi(t)$ satisfies Assumptions 2.4. 2.6, and 2.8. Consequently, the discrete convex hull property (Theorem 1.1) holds also for this larger class of problems.

In order to prove Theorem 4.1 for more general nonlinearities we need to ensure the solvability of the problem. This can e.g. be achieved either by restrictions on the right-hand side $f$ or by assuming the additional growth condition $\lim _{t \rightarrow \infty} \psi(t) / t=\infty$ for the non-linearity. We illustrate the first case in Remark 4.3 below by means of the mean curvature problem, i.e., $\psi(t)=\sqrt{1+t^{2}}$. The latter case includes for example all $N$-functions. For more information on $N$-functions and on finite element approximations for this kind of problems we refer to [DR07, DK08, BDK12].

Remark 4.3 (Mean curvature problem). We emphasize that also the discrete mean curvature problem fits into the setting of Section 2.2. To see this, we consider the corresponding energy

$$
\mathcal{J}(\boldsymbol{V}):=\int_{\Omega} \sqrt{1+|\nabla \boldsymbol{V}|^{2}} \mathrm{~d} x, \quad \boldsymbol{V} \in \mathbb{V}(\mathcal{T})^{m} .
$$

Obviously, the mapping $\Omega \times \mathbb{R}_{\geqslant} \ni(x, t) \mapsto F(x, t):=\sqrt{1+t^{2}}$ has linear growth and is monotone in $t$. Therefore, it satisfies Assumptions 2.4 and 2.8. Moreover, it is coercive and thanks to $\frac{d^{2}}{d t^{2}} \sqrt{1+t^{2}}=\frac{1}{\sqrt{1+t^{2}}}>0$ for all $t \geqslant 0$, it is strictly convex. This verifies Assumption 2.6 and consequently, minimisers $\boldsymbol{U} \in \boldsymbol{G}+\mathbb{V}_{0}(\mathcal{T})^{m}$ of the 
energy functional $\mathcal{J}$ in $\boldsymbol{G}+\mathbb{V}_{0}(\mathcal{T})^{m}, \boldsymbol{G} \in \mathbb{V}(\mathcal{T})$, satisfy the convex hull property Theorem 1.1.

Consider the scalar case $m=1$ and let $f \in L^{2}(\Omega), f \leqslant 0$ in $\Omega$. Let $G \in \mathbb{V}(\mathcal{T})$, according to $\mathrm{FV03}$, the energy

$$
\mathcal{J}_{f}(V):=\int_{\Omega} \sqrt{1+|\nabla V|^{2}}-f V \mathrm{~d} x, \quad V \in G+\mathbb{V}_{0}(\mathcal{T})
$$

is coercive if and only if there exists $\varepsilon>0$, such that

$$
\int_{\Omega} f V \mathrm{~d} x \leqslant(1-\varepsilon) \int_{\Omega}|\nabla V| \mathrm{d} x \quad \text { for all } V \in \mathbb{V}_{0}(\mathcal{T}) .
$$

Under this condition Theorem 4.1 directly applies to minimisers of the energy defined in 4.4.

4.2. Problems Involving Lower Order Terms. In this section we investigate non-linear problems involving lower order terms in form of lumped masses. Let $\mathcal{T}$ be a conforming triangulation of $\Omega$ and $\boldsymbol{G} \in \mathbb{V}(\mathcal{T})^{m}, m \in \mathbb{N}$. For $1<p, q<\infty$ let the energy

$$
\mathcal{J}(\boldsymbol{V}):=\int_{\Omega} \frac{1}{p}|\nabla \boldsymbol{V}|^{p}+\frac{1}{q}|\boldsymbol{V}|^{q} \mathrm{~d} x \quad \boldsymbol{V} \in \mathbb{V}(\mathcal{T})^{m}
$$

serve as our prototype. Obviously, there exists a unique minimiser of $\mathcal{J}$ in $\boldsymbol{G}+$ $\mathbb{V}_{0}(\mathcal{T})^{m}$.

In order to prove a convex hull property (Theorem 1.1) in the spirit of Section 3 . we would need an analogue of Lemma 3.2 for function values. Unfortunately, in general we do not have $|\boldsymbol{V}| \geqslant\left|\mathcal{P}_{K} \boldsymbol{V}\right|$ point-wise in $\Omega$. One can construct examples where the discrete maximum principle is violated even in one dimension; see e.g. BKK08. However, for $p=q=2$ it is well known that maximum principles hold for sufficiently small mesh-sizes if the mesh is strictly acute; see e.g. CR73, BKK08.

This additional assumptions can be avoided by mass-lumping, i.e., instead of a minimiser of 4.5 we look for a minimiser of the modified energy

$$
\mathcal{J}_{\ell}(\boldsymbol{V}):=\int_{\Omega} \frac{1}{p}|\nabla \boldsymbol{V}|^{p} \mathrm{~d} x+\sum_{z \in \mathcal{N}} \frac{\left|\operatorname{supp}\left(\phi_{z}\right)\right|}{n+1} \frac{1}{q}|\boldsymbol{V}(z)|^{q},
$$

which is coercive and strictly convex. Hence there exists a unique $\boldsymbol{U} \in G+\mathbb{V}_{0}(\mathcal{T})^{m}$ minimising $\mathcal{J}_{\ell}$ in $\boldsymbol{G}+\mathbb{V}_{0}(\mathcal{T})^{m}$. This minimiser satisfies the following convex hull property.

Theorem 4.4. Let the conforming triangulation $\mathcal{T}$ of $\Omega$ be non-obtuse and let $\boldsymbol{U} \in \boldsymbol{G}+\mathbb{V}_{0}(\mathcal{T})^{m}$ be the unique minimiser of the energy $\mathcal{J}_{\ell}$ in $\boldsymbol{G}+\mathbb{V}_{0}(\mathcal{T})^{m}$.

Then we have the max hull property

$$
\boldsymbol{U}(\Omega) \subset \operatorname{conv} \text { hull }(\boldsymbol{U}(\partial \Omega) \cup\{0\}) .
$$

Proof. Thanks to $K:=$ conv hull $(\boldsymbol{G}(\partial \Omega) \cup\{0\})$ we can choose $z=0$ in Lemma 3.1 and observe that

$$
0 \geqslant\left|\Pi_{K} x\right|^{2}-x \cdot \Pi_{K} x \geqslant\left|\Pi_{K} x\right|^{2}-|x|\left|\Pi_{K} x\right| \text { for all } x \in \mathbb{R}^{m} .
$$

Consequently we have

$$
\left|\Pi_{K} \boldsymbol{U}(z)\right| \leqslant|\boldsymbol{U}(z)| \quad \text { for all } z \in \mathcal{N} .
$$

Combining this with Lemma 3.2 , we obtain

$$
\mathcal{J}_{\ell}\left(\mathcal{P}_{K} \boldsymbol{U}\right) \leqslant \mathcal{J}_{\ell}(\boldsymbol{U})
$$

Recalling that the minimiser $\boldsymbol{U}$ is unique in $\boldsymbol{G}+\mathbb{V}_{0}(\mathcal{T})^{m}$ we have $\boldsymbol{U}=\mathcal{P}_{K} \boldsymbol{U}$. Hence we can conclude with $(3.3 \mathrm{a})$ that $\boldsymbol{U}(\Omega)=\left(\mathcal{P}_{K} \boldsymbol{U}\right)(\Omega) \subset K$. This proves the assertion. 
Remark 4.5. In the scalar case $m=1$ the minimiser $U \in G+\mathbb{V}_{0}(\mathcal{T})$ of the energy

$$
\mathcal{J}_{\ell}(V):=\int_{\Omega} \frac{1}{p}|\nabla V|^{p}-f V \mathrm{~d} x+\sum_{z \in \mathcal{N}} \frac{\left|\operatorname{supp}\left(\phi_{z}\right)\right|}{n+1} \frac{1}{q}|V(z)|^{q},
$$

with $f \leqslant 0$ satisfies the discrete maximum principle

$$
\max U(\Omega) \leqslant \max \{0, \max U(\partial \Omega)\} .
$$

This claim is an easy consequence of the above arguments combined with (4.3).

4.3. Strong Convex Hull Property. In some situations we have a strong version of the convex hull property. For scalar functions this is well known as the strong maximum principle: if a subharmonic function attains a global maximum in the interior of the domain $\Omega$, then the functions must be constant (on the connected component).

We will introduce in this section a discrete strong convex hull property. To this end we need that the conforming triangulation $\mathcal{T}$ of $\Omega$ is acute: A simplex $T \in \mathcal{T}$ is called acute if the angles between any two of its sides are less than $\frac{\pi}{2}$. Similar to Definition 2.1, we can formulate this definition also in terms of the Lagrange basis.

Definition 4.6 (acuteness). Let $\mathcal{T}$ be a conforming triangulation of $\Omega$. We call an $n$-simplex $T \in \mathcal{T}$ acute if

$$
\nabla \phi_{z \mid T} \cdot \nabla \phi_{y \mid T}<0 \quad \text { for all } z, y \in T \cap \mathcal{N} \text { with } z \neq y .
$$

The conforming triangulation $\mathcal{T}$ of $\Omega$ is called acute if all simplexes $T \in \mathcal{T}$ are acute.

Let $K \subset \mathbb{R}^{m}$ be a non-empty, convex, and closed set. Then we call $x \in K$ an extreme point if it is no element of a line segment of two different points of $K$. By $\operatorname{extr}(K)$ we denote the set of extreme points of $K$. It is well known that if $K$ is additionally bounded, we have conv hull $(\operatorname{extr}(K))=\operatorname{conv} \operatorname{hull}(K)$.

We assume the following additional condition on our mesh.

Assumption 4.7. We assume that $\mathcal{T}$ is a conforming triangulation of $\Omega$ such that for every $T \in \mathcal{T}$, we have $T \cap \mathcal{N}_{0} \neq \varnothing$.

In other words, every simplex of the triangulation $\mathcal{T}$ has at least one interior vertex. As a consequence, we have that every vertex of the boundary is in the support of one of the interior Lagrange basis functions. Assumption 4.7 is not a severe restriction.

We can now state the discrete strong convex hull property for discrete $p$-harmonic functions, i.e., solutions of (4.1).

Theorem 4.8. Let the acute conforming triangulation $\mathcal{T}$ of $\Omega$ satisfy Assumption 4.7. Let $\boldsymbol{U} \in \boldsymbol{G}+\mathbb{V}_{0}(\mathcal{T})^{m}$, be discretely p-harmonic with boundary values $\boldsymbol{G} \in$ $\mathbb{V}(\mathcal{T})^{m}$, i.e., $\boldsymbol{U}$ is a solution of (4.1).

If $\boldsymbol{U}\left(z_{0}\right) \in \operatorname{extr}\left(\operatorname{conv} \operatorname{hull}(\boldsymbol{U}(\Omega))\right.$ for some $z_{0} \in \Omega$, then $\boldsymbol{U}$ is constant.

Before we get to the proof of Theorem 4.8 we need a local version of this result.

Lemma 4.9. Let $\mathcal{T}$ be acute and let $\boldsymbol{U}$ be a solution of 4.1). Let $z_{0} \in \mathcal{N}_{0}$ and let $C:=\left(\mathcal{N} \backslash\left\{z_{0}\right\}\right) \cap \operatorname{supp} \phi_{z_{0}}$, i.e., $C$ is the set of neighbors of $z_{0}$. Then $\boldsymbol{U}\left(z_{0}\right) \in$ $\operatorname{extr}($ conv hull $(\boldsymbol{U}(C)))$ implies that $\boldsymbol{U}$ is constant on $\operatorname{supp} \phi_{z_{0}}$.

Proof. Let us assume that $\boldsymbol{U}$ is non-constant on $\operatorname{supp} \phi_{z_{0}}$. We have to prove that $\boldsymbol{U}\left(z_{0}\right) \notin \operatorname{extr}(\operatorname{conv} \operatorname{hull}(\boldsymbol{U}(C)))$. 
Since $z_{0} \in \mathcal{N}_{0}$, we have for every unit vector $\boldsymbol{e}_{j} \in \mathbb{R}^{m}, j=1, \ldots, m$, that $\boldsymbol{e}_{j} \phi_{z_{0}} \in \mathbb{V}_{0}(\mathcal{T})^{m}$. Therefore, it follows from 4.1 that

$$
\int_{\Omega}|\nabla \boldsymbol{U}|^{p-2} \nabla \boldsymbol{U}: \nabla\left(\boldsymbol{e}_{j} \phi_{z_{0}}\right) \mathrm{d} x=0 \quad \text { for all } j=1, \ldots, m .
$$

Thanks to the representation $\boldsymbol{U}=\sum_{y \in \mathcal{N}} \boldsymbol{U}(y) \phi_{y}$, we thus get

$$
\sum_{y \in \mathcal{N}} \boldsymbol{U}(y) \int_{\Omega}|\nabla \boldsymbol{U}|^{p-2} \nabla \phi_{y} \cdot \nabla \phi_{z_{0}} \mathrm{~d} x=0
$$

or equivalently

$$
\boldsymbol{U}\left(z_{0}\right) \underbrace{\int_{\Omega}|\nabla \boldsymbol{U}|^{p-2} \nabla \phi_{z_{0}} \cdot \nabla \phi_{z_{0}} \mathrm{~d} x}_{:=\beta_{0}}=\sum_{y \in C} \boldsymbol{U}(y) \underbrace{\int_{\Omega}|\nabla \boldsymbol{U}|^{p-2}\left(-\nabla \phi_{y} \cdot \nabla \phi_{z_{0}}\right) \mathrm{d} x}_{=: \beta_{y}} .
$$

By assumption $\boldsymbol{U}$ is non-constant on $\operatorname{supp} \phi_{z_{0}}$, hence $\beta_{0}>0$. On the other hand, since $\mathcal{T}$ is acute, we have $\beta_{y} \geqslant 0$ for all $y \in C$. Let $T \in \mathcal{T}, T \subset \operatorname{supp} \phi_{z_{0}}$ such that $\boldsymbol{U}_{\mid T} \not \equiv 0$. If $y \in \mathcal{N} \cap T, y \neq z_{0}$, then the acuteness of $T$ implies $\beta_{y}>0$. Thus at least two (more precisely: at least $n$ ) of the $\beta_{y}$ with $y \in C$ satisfy $\beta_{y}>0$.

The identity $\phi_{z_{0}}+\sum_{y \in C} \phi_{y}=1$ on $\operatorname{supp} \phi_{z_{0}}$ implies $\beta_{0}=\sum_{y \in C} \beta_{y}$. Therefore, defining $\lambda_{y}:=\beta_{y} / \beta_{z_{0}}$ yields $\boldsymbol{U}\left(z_{0}\right)=\sum_{y \in C} \lambda_{y} \boldsymbol{U}(y)$ with $\lambda_{y} \geqslant 0$ for $y \in C$ and $\sum_{y \in C} \lambda_{y}=1$. Consequently, at least two of the $\lambda_{y}$ with $z \in C$ satisfy $\lambda_{y}>0$ and hence $\boldsymbol{U}\left(z_{0}\right)$ cannot be an extreme point of conv hull $\{\boldsymbol{U}(y): y \in C\}$. In other words, we have $\boldsymbol{U}\left(z_{0}\right) \notin \operatorname{extr}(\operatorname{conv} \operatorname{hull}(\boldsymbol{U}(C)))$.

We can now prove Theorem 4.8

Theorem 4.8. Since $\boldsymbol{U}$ is piecewise linear and thanks to the properties of extremal points, it follows that there exists $\tilde{z}_{0} \in \mathcal{N}_{0}$ with $\boldsymbol{U}\left(z_{0}\right)=\boldsymbol{U}\left(\tilde{z}_{0}\right)$. Therefore, we may assume w.l.o.g. that $z_{0} \in \mathcal{N}_{0}$.

Suppose $\boldsymbol{U}\left(z_{0}\right) \in \operatorname{extr}\left(\operatorname{conv} \operatorname{hull}(\boldsymbol{U}(\Omega))\right.$ and let $C:=\left(\mathcal{N} \backslash\left\{z_{0}\right\}\right) \cap \operatorname{supp} \phi_{z_{0}}$ be the neighbors of $z_{0}$. Due to the convex hull property of $\boldsymbol{U}$ (see Theorem 1.1) we have that convhull $(\boldsymbol{U}(C)) \subset \operatorname{conv} \operatorname{hull}(\boldsymbol{U}(\partial \Omega))$. Since $\boldsymbol{U}\left(z_{0}\right)$ is an extreme point of conv hull $(\boldsymbol{U}(\partial \Omega))$ it must also be an extreme point of $\operatorname{extr}(\operatorname{conv} \operatorname{hull}(\boldsymbol{U}(C))$. Therefore, by Lemma 4.9, $\boldsymbol{U}$ is locally constant on $\operatorname{supp} \phi_{z_{0}}$.

A repetition of this argument shows that $\boldsymbol{U}$ is constant on its connected component, which is $\Omega$ itself. For this argument we need, that every simplex contains at least one vertex of $\mathcal{N}_{0}$.

Remark 4.10. We emphasize that 4.1) serves as a model problem and that Theorem 4.8 applies to more general non-linearities. For example, the strong convex hull property applies to the non-linear problems mentioned in Remark 4.2 if $\mathcal{J}$ is additionally Fréchet differentiable, i.e., if the minimising problem 1.1 can be equivalently formulated by a partial differential equation, the so-called Euler-Lagrange equation; compare with 1.2 .

\section{ACKNOWLEDGEMENTS:}

Part of this work was carried out during a stay of Christian Kreuzer at the Mathematical Institute of the University of Oxford. This stay was financed by the German Research Foundation DFG within the research grant KR 3984/1-1.

Last but not least, the authors want to thank Adrian Hirn for the valuable and inspiring discussions during his stay in Munich. 


\section{REFERENCES}

[BDK12] L. Belenki, L. Diening, and C. Kreuzer, Optimality of an adaptive finite element method for the p-Laplacian equation, IMA J. Numer. Anal. 32 (2012), no. 2, 484-510.

[BF02] M. Bildhauer and M. Fuchs, Partial regularity for a class of anisotropic variational integrals with convex hull property, Asymptot. Anal. 32 (2002), no. 3-4, 293-315.

[BKK08] J. H. Brandts, S. Korotov, and M. Křížek, The discrete maximum principle for linear simplicial finite element approximations of a reaction-diffusion problem, Linear Algebra Appl. 429 (2008), no. 10, 2344-2357.

[CR73] P. G. Ciarlet and P.-A. Raviart, Maximum principle and uniform convergence for the finite element method, Comput. Methods Appl. Mech. Engrg. 2 (1973), 17-31.

[DDS05] A. Drăgănescu, T. F. Dupont, and L. R. Scott, Failure of the discrete maximum principle for an elliptic finite element problem, Math. Comp. 74 (2005), no. 249, 1-23.

[DK08] L. Diening and C. Kreuzer, Convergence of an adaptive finite element method for the p-Laplacian equation, SIAM J. Numer. Anal. 46 (2008), no. 2, 614-638.

[DLM98] A. D'Ottavio, F. Leonetti, and C. Musciano, Maximum principle for vector-valued mappings minimizing variational integrals, Atti Sem. Mat. Fis. Univ. Modena 46 (1998), no. suppl., 677-683.

[DR07] L. Diening and M. Rǔžička, Interpolation operators in Orlicz-Sobolev spaces., Numer. Math. 107 (2007), no. 1, 107-129.

[FV03] F. Fierro and A. Veeser, On the a posteriori error analysis for equations of prescribed mean curvature, Math. Comp. 72 (2003), no. 244, 1611-1634.

[Hua11] W. Huang, Discrete maximum principle and a delaunay-type mesh condition for linear finite element approximations of two-dimensional anisotropic diffusion problems, NMTMA 4 (2011), no. 3, 319-334.

[JU05] A. Jüngel and A. Unterreiter, Discrete minimum and maximum principles for finite element approximations of non-monotone elliptic equations, Numer. Math. 99 (2005), no. 3, 485-508.

[KK05a] J. Karátson and S. Korotov, Discrete maximum principles for finite element solutions of nonlinear elliptic problems with mixed boundary conditions, Numer. Math. 99 (2005), no. 4, 669-698.

[KK05b] S. Korotov and M. Křížek, Global and local refinement techniques yielding nonobtuse tetrahedral partitions, Comput. Math. Appl. 50 (2005), no. 7, 1105-1113.

[KK11] Nonobtuse local tetrahedral refinements towards a polygonal face/interface, Appl. Math. Lett. 24 (2011), no. 6, 817-821.

[KKN01] S. Korotov, M. Křížek, and P. Neittaanmäki, Weakened acute type condition for tetrahedral triangulations and the discrete maximum principle, Math. Comp. 70 (2001), no. 233, 107-119.

[KQ95] M. Křížek and L. Qun, On diagonal dominance of stiffness matrices in 3D, East-West J. Numer. Math. 3 (1995), no. 1, 59-69.

[Let92] F. W. Letniowski, Three-dimensional Delaunay triangulations for finite element approximations to a second-order diffusion operator, SIAM J. Sci. Statist. Comput. 13 (1992), no. 3, 765-770.

[LH10] X. Li and W. Huang, An anisotropic mesh adaptation method for the finite element solution of heterogeneous anisotropic diffusion problems, J. Comput. Phys. 229 (2010), no. 21, 8072-8094.

[RS82] V. Ruas Santos, On the strong maximum principle for some piecewise linear finite element approximate problems of nonpositive type, J. Fac. Sci. Univ. Tokyo Sect. IA Math. 29 (1982), no. 2, 473-491.

[SF73] G. Strang and G. J. Fix, An analysis of the finite element method, Prentice-Hall Inc., Englewood Cliffs, N. J., 1973, Prentice-Hall Series in Automatic Computation.

[WZ12] J. Wang and R. Zhang, Maximum principles for P1-conforming finite element approximations of quasi-linear second order elliptic equations, SIAM J. Numer. Anal. 50 (2012), no. 2, 626-642. 
Lars Diening, Mathematisches Institut der Universität München, Theresienstrasse 39, D-80333 München, Germany

URL: http://www . mathematik. uni-muenchen.de/ ${ }^{\sim}$ diening

E-mail address: Lars.Diening@mathematik.uni-muenchen.de

Christian Kreuzer, Fakultät für Mathematik, Ruhr-Universität Bochum, UniverSitÄtsstrasse 150, D-44801 Bochum, Germany

URL: http://www.ruhr-uni-bochum.de/ffm/Lehrstuehle/Kreuzer/

E-mail address: christan.kreuzer@rub.de

Sebastian Schwarzacher, Mathematisches Institut der Universität München, ThereSienstrasse 39, D-80333 MÜnChen, Germany

URL: http://www.mathematik. uni-muenchen.de/ ${ }^{\text {schwarz/ }}$

E-mail address: sebastian.scharzacher@mathematik.uni-muenchen.de 